\title{
Evolutionary conservation of meiotic DSB proteins: more than just Spo11
}

\author{
Francesca Cole, ${ }^{1,4}$ Scott Keeney, ${ }^{2}$ and Maria Jasin ${ }^{1,3}$ \\ ${ }^{1}$ Developmental Program, Memorial Sloan-Kettering Cancer Center, New York, New York 10065, USA; ${ }^{2}$ Molecular Biology \\ Program, Howard Hughes Medical Institute, Memorial Sloan-Kettering Cancer Center, New York, New York 10065, USA
}

\begin{abstract}
Meiotic recombination is initiated by programmed DNA double-strand breaks (DSBs) generated by the Spo11 protein. In budding yeast, five other meiotic-specific proteins are also required for DSB formation, but, with rare exception, orthologs had not been identified in other species. In this issue of Genes \& Development, Kumar and colleagues (pp. 1266-1280) used a phylogenomic approach to identify two of these proteins across multiple clades, and confirmed that one of these, MEI4, is a functional ortholog in mouse.
\end{abstract}

Recombination during meiosis increases genetic diversity, but it can be argued that its central purpose is to promote accurate segregation of homologous chromosomes for the generation of haploid gametes (Hunter 2007). During meiosis, programmed DNA double-strand breaks (DSBs) are introduced throughout the genome, repair of which leads to interhomolog recombination. Thus, meiotic cells incur substantial self-inflicted DNA damage, which has few parallels. Meiotic DSB repair is remarkably robust, having co-opted and elaborated existing DSB repair mechanisms, which exist in every cell to repair spontaneous DNA damage.

Interhomolog recombination is essential during meiosis because it leads to the formation of crossovers (COs), which are required for the accurate disjunction of homologs during the reductional phase of meiosis (Fig. 1). COs, in conjunction with sister chromatid cohesion, provide the connections between homologs to allow them to align at the metaphase plate. Thus, as in mitotic divisions with sister chromatids, homolog pairs in meiosis are stably joined to allow them to be coordinately captured by both spindle poles (Fig. 1A). With the release of sister chromatid cohesion, recombinant homologs segregate to opposite poles (Fig. 1B), halving the genome content. A failure to form COs results in the inability of homolog pairs to align at the metaphase plate, preventing proper segregation (Fig. 1C,D). The complete absence of COs is

[Keywords: Meiosis; recombination; double-strand break]

Corresponding authors.

${ }^{3}$ E-MAIL jasinm@mskcc.org; FAX (212) 772-8410.

${ }^{4}$ E-MAIL colef@mskcc.org. FAX (212) 772-8410.

Article is online at http://www.genesdev.org/cgi/doi/10.1101/gad.1944710. a catastrophic event that can lead to cell death, whereas the absence of a CO from a single homolog pair often leads to aneuploidy, which is the leading cause of spontaneous miscarriage and developmental defects in humans (Hassold et al. 2007). The identification of proteins involved in meiotic DSB formation and repair are therefore critical for our understanding of homologous recombination and the causes of infertility. This perspective focuses on proteins involved in meiotic DSB formation, highlighting the recent discovery of the long-sought-after orthologs of the yeast Mei4 and Rec114 (Kumar et al. 2010).

\section{Meiotic recombination mechanisms}

Meiotic recombination mechanisms have been inferred primarily from studies in budding yeast (Bishop and Zickler 2004; Baudat and de Massy 2007; Hunter 2007; Keeney 2007). DSBs are introduced by the Spol1 protein, and once Spoll is removed from DNA, the 5' ends are resected to generate $3^{\prime}$ single-stranded tails (Fig. 2). The ssDNA is bound by RecA family members (Rad51 and Dmc1), creating nucleoprotein filaments for strand invasion of the homologous duplex (Chen et al. 2008; San Filippo et al. 2008). Repair synthesis occurs from the $3^{\prime}$ end in this displacement loop (D-loop). In the canonical DSB repair (DSBR) model, the other DNA end is "captured" by the D-loop, forming a double Holliday junction $(\mathrm{dHJ})$, which has been observed in molecular assays. Resolution of the $\mathrm{dHJ}$ generates COs, with reciprocal exchange of flanking markers. Noncrossovers (NCOs) contain a gene conversion tract without exchange of flanking markers, and theoretically could also be generated from resolution of dHJs. However, NCOs are formed primarily by a different mechanism, as evidenced by their distinct genetic requirements, intermediates, and timing. NCOs instead appear to arise from synthesis-dependent strand annealing (SDSA), in which the extended 3' end is expelled from the D-loop to anneal with the other end of the DSB (Paques and Haber 1999; McMahill et al. 2007).

In mitotically dividing cells, recombination reactions strongly favor use of the sister chromatid as the template for DSB repair (Paques and Haber 1999; Moynahan and Jasin 2010). During meiosis, in contrast, use of the homolog is favored over use of the sister chromatid, in keeping with the essential role that recombination plays 
Cole et al.

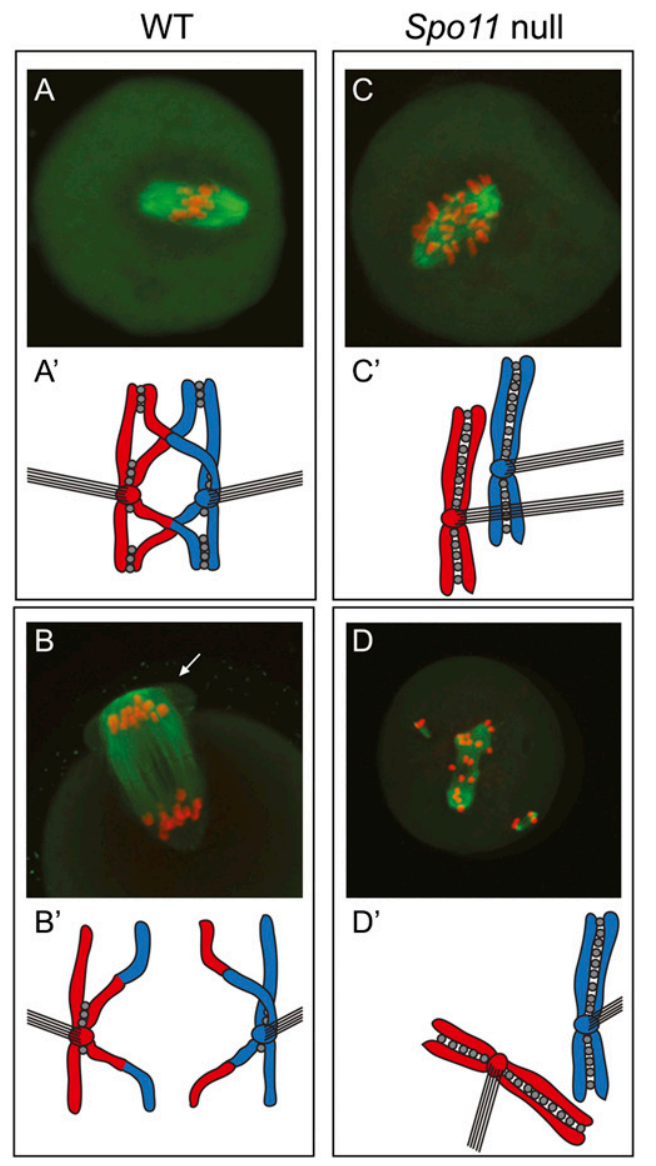

Figure 1. Interhomolog recombination is essential for proper chromosome segregation during meiosis I. Mouse oocytes were cultured in vitro and then stained for DNA (red) and $\beta$-tubulin (green). In oocytes from wild-type mice, chromosomes congress at the metaphase plate $(A)$, and homologs segregate at anaphase, accompanied by extrusion of the first polar body (arrow) (B). In Spo11-null oocytes, homologs are unconnected to each other such that bipolar attachment and chromosome congression at the metaphase plate are impossible. $(C)$ As a result, chromosomes are chaotically distributed along the spindle. $(D)$ Eventually, the spindle can break down, without oocytes proceeding through meiosis I. Images are courtesy of Monica Di Giacomo. Schematics of chromosome dynamics. In wild-type oocytes, the two homologs (red and blue) of this metacentric chromosome have undergone a $\mathrm{CO}$ on each chromosome arm. $\left(A^{\prime}\right)$ Sister chromatids are attached to each other by cohesion (gray circles), which holds the recombinant homologs at the metaphase plate. $\left(B^{\prime}\right)$ When cohesion is released at anaphase, homologs segregate to opposite poles. In Spo11-null mice, homologs are not connected because there are no COs. $\left(C^{\prime}, D^{\prime}\right)$ Thus, they attach independently of one another and frequently attach to the same pole. Bundles of lines represent spindle microtubules.

in connecting homologs to one another (Hunter 2007). A twist on the canonical DSBR model has been proposed recently, in which both $3^{\prime}$ ends of a DSB simultaneously invade different homologous duplexes, resulting in repair synthesis from both (Hunter 2007). The simultaneous invasion of two homologous duplexes is possible because recombination occurs at the four-chromatid stage (two sister chromatids for each homolog) (not shown in Fig. 2).
Thus, this model suggests that a single DSB could productively engage both the homolog and its sister chromatid, which provides an intriguing challenge to the view that there is a strict dichotomy between interhomolog and intersister recombination.

While each chromosome requires at least one $\mathrm{CO}$ to ensure accurate segregation in meiosis I, interhomolog NCOs are presumed to predominate in number compared with COs (Baudat and de Massy 2007; Martinez-Perez and Colaiacovo 2009). This is particularly true in mammals, where, for example, mouse spermatocytes are estimated to average $\sim 200-300$ DSBs and $\sim 23-24$ COs per meiosis, implying that NCOs outnumber COs by a factor of 10 to one. The excess of NCOs likely reflects a requirement for multiple interhomolog interactions to mediate pairing between homologs.

\section{DSB formation and Spo11 removal}

During meiosis, Spo11 catalyzes phosphodiester cleavage, but a suite of additional proteins-nine in budding yeast-is required for Spol1 to act. Furthermore, once DSB formation occurs, Spol1 is nucleolytically removed from DNA, requiring at least one additional factor. The remainder of this perspective will focus on these steps-DSB formation and Spol1 removal-and the proteins involved in the process.

\section{Spo11: a transesterase}

Spol1 was identified as the catalytic component required for meiotic DSB formation in yeast by two independent groups, both of which relied on the observation that unresected meiotic DSBs accumulate in a rad50S mutant (Bergerat et al. 1997; Keeney et al. 1997). In this mutant, a protein is covalently bound to the DNA ends, suggesting a transesterase mechanism for DSB formation, such as with topoisomerases, rather than simple hydrolytic cleavage of DNA. Homology between Spol1 and a type II archaeal topoisomerase, together with identification of a critical tyrosine residue for DSB formation, led to the prediction that Spol1 was the protein bound to DNA ends in the rad50S mutant (Bergerat et al. 1997). Direct demonstration of this prediction was simultaneously provided by purification of Spo11-DNA complexes (Keeney et al. 1997).

Spol1 cleaves DNA through a nucleolytic attack to generate a $5^{\prime}$ phosphotyrosyl bond and a 3' hydroxyl. Rather than simply reversing the reaction to reseal the DNA, as with topoisomerases, Spol1 is trapped on the DNA. By analogy with the archaeal topoisomerase, Spo11 functions as a homodimer in which each active site is made up of residues from both subunits (Nichols et al. 1999). In yeast, Spo11 apparently cleaves DNA to leave a 2-nucleotide (nt) 5' overhang (Liu et al. 1995), suggesting that the catalytic tyrosine from each subunit attacks adjacent base pairs on the two strands (Fig. 2A-E).

\section{Spo11 removal from DNA}

For DNA end resection to proceed, Spol1 must be removed from the DNA ends. Removal occurs by endonucleolytic 


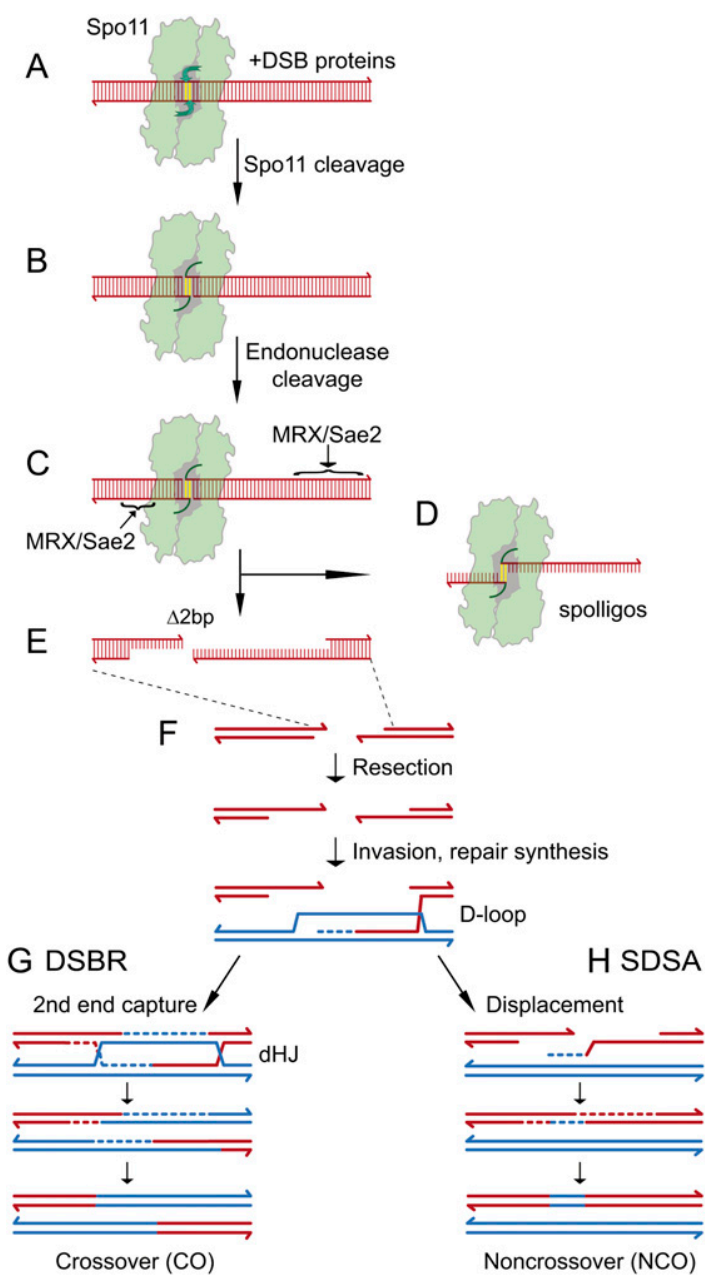

Figure 2. Spol1-mediated DSBs and homologous recombination pathways. (A) The Spoll homodimer (green and gray shading) is transparent in order to see the duplex DNA underneath (red). Each Spol1 monomer attacks the phosphodiester backbone of the DNA at adjacent base pairs (yellow lines), using a catalytic tyrosine residue with assistance from a metal binding pocket (green arrows). The outline of the structure is based on the structure of the Methanococcus jannaschi TopoVIA (Nichols et al. 1999). (B) After DSB formation, Spol1 is covalently linked (green arcs) to the 5' ends of DNA, leaving 3' hydroxyls and nicks offset by 2 base pairs (bp) (yellow lines). (C) MRX/Sae2 endonucleolytically cleaves DNA asymmetrically (brackets) (Neale et al. 2005). (D) The released Spo11 dimer is covalently attached to two oligonucleotides of different lengths, sometimes called "spolligos." $(E)$ The resulting DSB in the DNA has short 3' singlestranded tails of two different lengths. Note that the DSB is associated with a 2-bp gap because Spo11 cleaved at $5^{\prime}$ recessed phosphates. $(F-H)$ An expanded view of the DNA shown in $A-E$. (F) Further 5'-to-3' resection of the DNA ends, followed by strand invasion of the intact homologous duplex (blue) to form a D-loop. The $3^{\prime}$ end serves as a primer to initiate repair synthesis using the homologous DNA as template (dotted lines). At this point, the D-loop intermediate can proceed by either of two pathways, although it may be more stable in the DSBR pathway. $(G)$ The DSBR pathway. The other 3' end of the DSB is captured to form a dHJ. Resolution of the $\mathrm{dHJ}$ primarily forms COs. $(H)$ The SDSA pathway. The invading 3 ' strand is displaced and anneals to the other $3^{\prime}$ end of the DSB to primarily form NCOs. cleavage several nucleotides downstream from the 5' end, releasing each Spo11 monomer bound to an oligonucleotide (Neale et al. 2005). In some organisms, Spo11associated oligonucleotides come in two size ranges, which in mouse are $\sim 12-26 \mathrm{nt}$ and $28-34 \mathrm{nt}$. Time courses in budding yeast demonstrate similar kinetics and levels for both size ranges, raising the possibility that each DSB is processed to release one Spo11 monomer with a small oligonucleotide and one with a long oligonucleotide (Neale et al. 2005). Asymmetric cleavage is an attractive mechanism to differentiate the two DNA ends of a DSB for the next step of recombination: strand invasion (Neale et al. 2005).

In budding and fission yeasts, endonucleolytic removal of Spo11 requires the nuclease activity of the Mre11Rad50-Xrs2 (MRX) complex and its functional partner, Sae2/Ctp1 (Hunter 2007; Keeney 2007; Hartsuiker et al. 2009; Milman et al. 2009). These proteins play a more general role in the resection of mitotic DSBs (Mimitou and Symington 2009), as well as having other functions, and in mice are essential during early development, precluding a determination of their role in mammalian meiosis.

\section{Other proteins required for meiotic DSB formation}

In several organisms, genetic screens have defined components required for meiotic DSB formation (Keeney 2007). The most well-delineated pathway is that used by budding yeast where at least nine other proteins in addition to Spo11 are required (Hunter 2007; Keeney 2007). Three of these comprise MRX, revealing a role for this complex in both promoting Spol1-generated DSBs and removing covalently bound Spol1 from DNA. The complex interacts with chromatin in a Spo11-independent manner, although its function in promoting DSB formation is unclear. In Caenorhabditis elegans as well, Rad-50 and Mre-11 are required for DSB formation in otherwise wild-type meiosis /Chin and Villeneuve 2001; Hayashi et al. 2007), but the MRX requirement in meiotic DSB formation is not conserved in other organisms that have been examined, despite the fact that Rad50 and Mre11 are present throughout evolution (Keeney 2007).

The remaining Saccharomyces cerevisiae proteins required for DSB formation are meiotic-specific for expression (Mei4, Rec102, Rec104, and Rec114), splicing (Mer2), or nuclear localization (Ski8) (Keeney 2007). Physical interactions between these proteins, their subnuclear localization, and the genetic dependence for that localization have defined a series of subgroups. Spo11/Ski8 associates with DSB hot spots throughout the genome on the chromatin loops, and can interact directly with a subgroup containing Rec102/Rec104, which is localized near hot spots and also on chromatin loops (Arora et al. 2004; Kee et al. 2004; Prieler et al. 2005). These two subgroups are mutually dependent on each other for appropriate subnuclear localization. Mei4, Rec114, and Mer2 form another distinct subgroup that is thought to interact via Rec114 with the Rec102/Rec104 subgroup (Maleki et al. 2007). Finally, Xrs2 and Mer2 form a robust 
Cole et al.

interaction dependent on specific post-translational modifications of Mer2 (Arora et al. 2004; Henderson et al. 2006). Intriguingly, those same modifications are required for Mer2 association with Rec114 (Henderson et al. 2006; Maleki et al. 2007).

Of the seven meiotic-specific proteins required for DSB formation in budding yeast, only Spol1 has obvious evolutionary and functional conservation across multiple clades (Keeney 2007). Indeed, in mammals, only SPO11 has been identified from this group (Baudat et al. 2000; Romanienko and Camerini-Otero 2000). (MEI1, another protein required for DSB formation in mice, was discovered using a forward genetic screen [Libby et al. 2003], and appears to be conserved in Arabidopsis thaliana [De Muyt et al. 2007] but not in fungi.) This lack of clear orthologs for the $S$. cerevisiae proteins required for DSB formation could mean that these proteins do not exist in other clades, they exist but are not well-conserved, or, as is the case for Ski8, orthologs exist but are not required for DSB formation outside of fungi (Jolivet et al. 2006). Thus, although it is clear that Spol1-mediated DSBs initiate meiotic recombination in many organisms, the available data seemed to suggest that the process is fundamentally distinct in different organisms. Kumar et al. (2010) provide compelling evidence to the contrary, arguing that the same players are involved, but in disguise.

\section{Evolutionary conservation of the DSB proteins MEI4 and REC114}

In this issue of Genes \& Development, Kumar et al. (2010) used a phylogenomic-oriented homology search and alignment along with secondary structure prediction analysis (Bourbon 2008) to screen the sequenced genomes of budding yeasts (Saccharomycetales) and fission yeasts (Schizosaccharomycetales) for orthologs to Mei4 and Rec114. Once orthologs were identified, primary structure alignments allowed Kumar et al. (2010) to identify several short stretches of more highly conserved sequence, termed signature sequence motifs (SSMs), several of which had been observed previously within Saccharomyces species (Maleki et al. 2007). Unlike prior analyses, however, Kumar et al. (2010) successfully leveraged these findings to identify Mei4 and Rec114 orthologs in other species, using the SSMs for iterative alignment while incorporating parameters for the relative spacing between SSMs, conservation of key amino acids, and secondary structure characteristics. Notably, orthologs were identified in humans and mice, but interestingly, not in several organisms that play key roles in meiosis research, including flies, worms, and Sordariales.

As the SSMs make up only $\sim 20 \%$ of both proteins, the overall sequence identity and similarity is quite low. For example, in mice, the MEI4 and REC114 orthologs have only $8 \%$ and $6 \%$ identity with budding yeast, respectively. Given this poor sequence conservation, it is perhaps not surprising that this analysis also determined that previously identified proteins with meiotic functions were actually unrecognized orthologs of Mei4 and Rec114. For example, fission yeast Rec24 and Arabidop- sis $\operatorname{Prd} 2$, both of which are required for DSB formation, are Mei4 orthologs.

\section{Mouse MEI4 is a bona fide DSB protein}

To conclusively demonstrate that these proteins are essential for DSB formation requires the analysis of mutant phenotypes. Kumar et al. (2010) therefore targeted the mouse Mei4 gene for disruption. Satisfyingly, Mei4-null mice showed the same phenotypes as Spo11-null mice (Baudat et al. 2000; Romanienko and Camerini-Otero 2000). Namely, mice were viable but infertile, with males exhibiting small testes and epididymides devoid of sperm. Spermatocytes arrested during early meiotic prophase and showed high levels of apoptosis. Ovaries were also severely abnormal, showing a nearly complete loss of follicles at adulthood. In contrast, ovaries from juveniles had normal numbers of growing follicles, evidence that a portion of abnormal oocytes in these mice could escape attrition because of the lack of Spol1-mediated DNA damage (Di Giacomo et al. 2005).

Cytological analysis of meiotic chromosomes in spermatocytes also showed a similar phenotype as that observed for Spo11-null mice, including the absence of markers for DSB formation, including RAD51 and DMC1 foci (Baudat et al. 2000; Romanienko and Camerini-Otero 2000). The formation of $\gamma \mathrm{H} 2 \mathrm{AX}$ was also dramatically reduced in early meiotic prophase, indicative of a lack of DSB formation (Mahadevaiah et al. 2001), and, as has also been seen in Spo11-null mice (Barchi et al. 2005), the formation of a pseudosex body with accumulated $\gamma \mathrm{H} 2 \mathrm{AX}$ was observed later in meiotic prophase. Finally, Kumar et al. (2010) provide evidence that mouse MEI4 and REC114 proteins interact physically with one another, as in yeasts (Arora et al. 2004; Li et al. 2006; Steiner et al. 2010), arguing for functional conservation of Rec114 as well. Thus, Mei4-and probably Rec114-is evolutionarily conserved among yeast, plants, and mice, indicating that these proteins and their roles in meiotic recombination are almost a billion years old.

\section{Rapid evolution of meiotic DSB proteins}

Spo11 has evolutionarily constrained catalytic motifs that maintain a minimal level of sequence conservation and thus allow ready identification of orthologs throughout eukaryotic lineages, even though overall conservation is fairly low (e.g., $23 \%$ identity between yeast and mice) (Keeney 2007; Malik et al. 2007). In contrast, orthologs of the other meiosis-specific DSB proteins from $S$. cerevisiae have been very difficult (Mei4 and Rec114) or thus far impossible (Mer2, Rec102, and Rec104) to identify in other clades. In fact, these DSB proteins are among the most rapidly diverging of all cellular proteins (Richard et al. 2005; Keeney 2007). For example, Rec114 homologs in $S$. cerevisiae and its very close relative Saccharomyces paradoxus have only $73 \%$ amino acid identity (Keeney 2007), much lower than even the overall nucleotide sequence identity between coding regions in these two species $(>88 \%)$. 
Why are meiotic DSB genes so highly diverged? One possibility is that this divergence reflects a tendency of reproductive genes to show more rapid evolution to block production of less vigorous hybrids due to incipient species (Swanson and Vacquier 2002). Another possibility is suggested by consideration of exciting recent studies by several groups (Neale 2010), including the same de Massy group (Baudat et al. 2010) featured in this perspective. These studies suggest that PRDM9, a histone H3-Lys 4 trimethyltransferase, binds to specific DNA sequences at many sites in the genome, targeting them for DSB formation. The zinc finger DNA-binding domain of PRDM9 undergoes remarkably rapid evolution, thereby conferring similarly rapid evolution on the array of sites to which SPO11-dependent DSB formation is targeted. Because DNA sequences are copied from the intact DNA duplex during DSB repair (Fig. 2), there is a net transfer of genetic information during recombination in favor of the unbroken chromosome. As a consequence of this biased gene conversion, hot spots tend to extinguish themselves over long time scales (a phenomenon sometimes referred to as the "hot spot paradox"). The ability of rapid changes in PRDM9 binding specificity to alter the landscape of meiotic recombination may confer a degree of evolutionary plasticity that counters hot spot extinction. Although the rapid divergence of PRDM9 is associated primarily with its DNA-binding domain, concerted coadaptation of other DSB proteins in response to $\operatorname{Prdm} 9$ mutation is conceivable. More generally, however, even for organisms (like budding yeast) that do not use an obviously PRDM9like system involving a dedicated DNA sequence-specific DSB landscape architect, PRDM9 nevertheless provides a precedent for a rapidly evolving DNA hot spot determinant. Thus, the observed rapid changes of more integral components of the recombination initiation machinery, such as Mei4 and even Spol1 itself, may be a reflection of the same evolutionary pressures.

\section{Insights from localization of MEI4 to chromosome axes in mice}

Cytological data in many organisms demonstrate that DSB repair occurs in close juxtaposition with the axes (Kleckner 2006), but in S. cerevisiae (and possibly in other organisms), DSB hot spots are located predominantly within the chromatin loops that emanate from axes rather than within the sequences thought to anchor the DNA to axes (Blat et al. 2002). This apparent paradox has led to the proposal that recombination occurs within multiprotein complexes that tether DSB ends to the axes; such "tethered loop-axis complexes" are envisioned to provide a means to coordinate the nanometer-scale DNA events of recombination with the micrometer-scale structural rearrangements of meiotic chromosomes (Blat et al. 2002; Kleckner 2006). In principle, tethering could occur at any of a number of points before, during, or after DSB formation (Kleckner 2006), but the protein-protein interactions that coordinate DSB formation with chromosome axes remain unknown.

In this context, it is thus intriguing that Kumar et al. (2010) found MEI4 associated with the chromosome axes in a SPO11-independent manner. Rec7 (the Schizosaccharomyces pombe homolog of Rec114) has also been shown to be axis-associated (Lorenz et al. 2006). Mei4 and Rec114 in S. cerevisiae have been reported to at least partially localize to chromatin loops, but available data do not exclude an axis-associated fraction of these proteins (Maleki et al. 2007). To explain why mouse MEI4 is located at the axes prior to DSB formation and is completely required for DSB formation, Kumar et al. (2010) propose that DSBs are formed after the loop becomes tethered to the axis, with MEI4 providing part of the connection between the axis and the DNA sequence that will be broken. Additional data from yeast suggest further specific elements of this connection: Mei4 and Rec114 interact with Rec102/Rec104, which themselves interact with Spo11/Ski8 and are localized to chromatin loops at or near DSB hot spots, respectively (Arora et al. 2004; Kee et al. 2004; Prieler et al. 2005; Maleki et al. 2007). Furthermore, $S$. cerevisiae Rec114 and Mei4 proteins interact with Mer2, whose activities are influenced by cell cycle regulatory kinases Cdc28-Clb5/Clb6 and Cdc7Dbf4 (Henderson et al. 2006; Sasanuma et al. 2008; Wan et al. 2008). Many of these interactions are also likely to be dynamic, such that, once a DSB is formed, the complexes rearrange and/or disassociate to allow repair proteins to mediate subsequent steps, or even to prevent multiple DSBs from occurring in close proximity. Such transience may explain the lack of colocalization between MEI4 and RAD51, DMC1, or RPA in mice (Kumar et al. 2010).

In this view, the complex network of interactions among the DSB proteins allows for regulation of DSB formation both temporally (with respect to meiotic progression) and spatially (with respect to axis-loop organization). As Mer2, Rec102, and Rec104 are key components of this network in budding yeast, it remains an important challenge to identify their orthologs (or functional substitutes) in mammals, whether through similar phylogenomic techniques or via interaction with MEI4 and REC114. In any case, the newly revealed evolutionary conservation provided by the studies of Kumar et al. (2010) heightens interest in more detailed mechanistic investigation of these proteins and their functions in connecting DSB formation to chromosome axes.

\section{Acknowledgments}

We thank Monica Di Giacomo (EMBL, Monterotondo) for permission to use the oocyte images; Franz Klein (University of Vienna), Yufuko Akamatsu (MSKCC), Andrew Bergemann (Mount Sinai School of Medicine), and Michael Lichten (NIH) for discussions; and Jim Dowdle (MSKCC) for coining the word "spolligos." This work was supported by a Ruth L. Kirschstein National Research Service Award F32HD51392 (to F.C.), and National Institutes of Health Grants R01HD40916 (to M.J., S.K.) and R01HD53855 (to S.K., M.J.).

\section{References}

Arora C, Kee K, Maleki S, Keeney S. 2004. Antiviral protein Ski8 is a direct partner of Spol1 in meiotic DNA break formation, 
independent of its cytoplasmic role in RNA metabolism. Mol Cell 13: 549-559.

Barchi M, Mahadevaiah S, Di Giacomo M, Baudat F, de Rooij DG, Burgoyne PS, Jasin M, Keeney S. 2005. Surveillance of different recombination defects in mouse spermatocytes yields distinct responses despite elimination at an identical developmental stage. Mol Cell Biol 25: 7203-7215.

Baudat F, de Massy B. 2007. Regulating double-stranded DNA break repair towards crossover or non-crossover during mammalian meiosis. Chromosome Res 15: 565-577.

Baudat F, Manova K, Yuen JP, Jasin M, Keeney S. 2000. Chromosome synapsis defects and sexually dimorphic meiotic progression in mice lacking Spo11. Mol Cell 6: 989-998.

Baudat F, Buard J, Grey C, Fledel-Alon A, Ober C, Przeworski M, Coop G, de Massy B. 2010. PRDM9 is a major determinant of meiotic recombination hotspots in humans and mice. Science 327: 836-840.

Bergerat A, de Massy B, Gadelle D, Varoutas PC, Nicolas A, Forterre P. 1997. An atypical topoisomerase II from Archaea with implications for meiotic recombination. Nature 386: 414-417.

Bishop DK, Zickler D. 2004. Early decision: Meiotic crossover interference prior to stable strand exchange and synapsis. Cell 117: 9-15.

Blat Y, Protacio RU, Hunter N, Kleckner N. 2002. Physical and functional interactions among basic chromosome organizational features govern early steps of meiotic chiasma formation. Cell 111: 791-802.

Bourbon HM. 2008. Comparative genomics supports a deep evolutionary origin for the large, four-module transcriptional mediator complex. Nucleic Acids Res 36: 3993-4008.

Chen Z, Yang H, Pavletich NP. 2008. Mechanism of homologous recombination from the RecA-ssDNA/dsDNA structures. Nature 453: 489-494.

Chin GM, Villeneuve AM. 2001. C. elegans mre-11 is required for meiotic recombination and DNA repair but is dispensable for the meiotic G(2) DNA damage checkpoint. Genes Dev 15: 522-534.

De Muyt A, Vezon D, Gendrot G, Gallois JL, Stevens R, Grelon M. 2007. AtPRD1 is required for meiotic double strand break formation in Arabidopsis thaliana. EMBO J 26: 4126-4137.

Di Giacomo M, Barchi M, Baudat F, Edelmann W, Keeney S, Jasin M. 2005. Distinct DNA-damage-dependent and -independent responses drive the loss of oocytes in recombination-defective mouse mutants. Proc Natl Acad Sci 102: 737-742.

Hartsuiker E, Mizuno K, Molnar M, Kohli J, Ohta K, Carr AM. 2009. Ctp1CtIP and Rad32Mre11 nuclease activity are required for Rec12Spo11 removal, but Rec12Spo11 removal is dispensable for other MRN-dependent meiotic functions. Mol Cell Biol 29: 1671-1681.

Hassold T, Hall H, Hunt P. 2007. The origin of human aneuploidy: Where we have been, where we are going. Hum Mol Genet 16: R203-R208. doi: 10.1093/hmg/ddm243.

Hayashi M, Chin GM, Villeneuve AM. 2007. C. elegans germ cells switch between distinct modes of double-strand break repair during meiotic prophase progression. PLoS Genet 3: e191. doi: 10.1371/journal.pgen.0030191.

Henderson KA, Kee K, Maleki S, Santini PA, Keeney S. 2006. Cyclin-dependent kinase directly regulates initiation of meiotic recombination. Cell 125: 1321-1332.

Hunter N. 2007. Meiotic recombination. In Molecular genetics of recombination (ed. A Aguilera, R Rothstein), pp. 381-442. Springer-Verlag, Berlin Heidelberg.

Jolivet S, Vezon D, Froger N, Mercier R. 2006. Non conservation of the meiotic function of the Ski8/Rec103 homolog in Arabidopsis. Genes Cells 11: 615-622.
Kee K, Protacio RU, Arora C, Keeney S. 2004. Spatial organization and dynamics of the association of Rec102 and Rec104 with meiotic chromosomes. EMBO I 23: 1815-1824.

Keeney S. 2007. Spo11 and the formation of DNA double-strand breaks in meiosis. In Recombination and meiosis (ed. $\mathrm{R}$ Egel, D-H Lankenau), pp. 81-123. Springer-Verlag, Berlin Heidelberg.

Keeney S, Giroux CN, Kleckner N. 1997. Meiosis-specific DNA double-strand breaks are catalyzed by Spo11, a member of a widely conserved protein family. Cell 88: 375-384.

Kleckner N. 2006. Chiasma formation: Chromatin/axis interplay and the role(s) of the synaptonemal complex. Chromosoma 115: 175-194.

Kumar R, Bourbon H-M, de Massy B. 2010. Functional conservation of Mei4 for meiotic DNA double-strand break formation from yeasts to mice. Genes Dev (this issue). doi: 10.1101/ gad.571710.

Li J, Hooker GW, Roeder GS. 2006. Saccharomyces cerevisiae Mer2, Mei4 and Rec114 form a complex required for meiotic double-strand break formation. Genetics 173: 19691981.

Libby BJ, Reinholdt LG, Schimenti JC. 2003. Positional cloning and characterization of Meil, a vertebrate-specific gene required for normal meiotic chromosome synapsis in mice. Proc Natl Acad Sci 100: 15706-15711.

Liu J, Wu TC, Lichten M. 1995. The location and structure of double-strand DNA breaks induced during yeast meiosis: Evidence for a covalently linked DNA-protein intermediate. EMBO J 14: 4599-4608.

Lorenz A, Estreicher A, Kohli J, Loidl J. 2006. Meiotic recombination proteins localize to linear elements in Schizosaccharomyces pombe. Chromosoma 115: 330-340.

Mahadevaiah SK, Turner JM, Baudat F, Rogakou EP, de Boer P, Blanco-Rodriguez J, Jasin M, Keeney S, Bonner WM, Burgoyne PS. 2001. Recombinational DNA double-strand breaks in mice precede synapsis. Nat Genet 27: 271-276.

Maleki S, Neale MJ, Arora C, Henderson KA, Keeney S. 2007. Interactions between Mei4, Rec114, and other proteins required for meiotic DNA double-strand break formation in Saccharomyces cerevisiae. Chromosoma 116: 471-486.

Malik SB, Ramesh MA, Hulstrand AM, Logsdon JM Jr. 2007. Protist homologs of the meiotic Spoll gene and topoisomerase VI reveal an evolutionary history of gene duplication and lineage-specific loss. Mol Biol Evol 24: 2827-2841.

Martinez-Perez E, Colaiacovo MP. 2009. Distribution of meiotic recombination events: Talking to your neighbors. Curr Opin Genet Dev 19: 105-112.

McMahill MS, Sham CW, Bishop DK. 2007. Synthesis-dependent strand annealing in meiosis. PLoS Biol 5: e299. doi: 10.1371/ journal.pbio.0050299.

Milman N, Higuchi E, Smith GR. 2009. Meiotic DNA doublestrand break repair requires two nucleases, MRN and Ctp1, to produce a single size class of Rec12 (Spol1)-oligonucleotide complexes. Mol Cell Biol 29: 5998-6005.

Mimitou EP, Symington LS. 2009. Nucleases and helicases take center stage in homologous recombination. Trends Biochem Sci 34: 264-272.

Moynahan ME, Jasin M. 2010. Mitotic homologous recombination maintains genomic stability and suppresses tumorigenesis. Natl Rev 11: 196-207.

Neale MJ. 2010. PRDM9 points the zinc finger at meiotic recombination hotspots. Genome Biol 11: 104. doi: 10.1186/ gb-2010-11-2-104.

Neale MJ, Pan J, Keeney S. 2005. Endonucleolytic processing of covalent protein-linked DNA double-strand breaks. Nature 436: 1053-1057. 
Nichols MD, DeAngelis K, Keck JL, Berger JM. 1999. Structure and function of an archaeal topoisomerase VI subunit with homology to the meiotic recombination factor Spo11. EMBO J 18: 6177-6188.

Paques F, Haber JE. 1999. Multiple pathways of recombination induced by double-strand breaks in Saccharomyces cerevisiae. Microbiol Mol Biol Rev 63: 349-404.

Prieler S, Penkner A, Borde V, Klein F. 2005. The control of Spo11's interaction with meiotic recombination hotspots. Genes Dev 19: 255-269.

Richard GF, Kerrest A, Lafontaine I, Dujon B. 2005. Comparative genomics of hemiascomycete yeasts: Genes involved in DNA replication, repair, and recombination. Mol Biol Evol 22: 1011-1023.

Romanienko PJ, Camerini-Otero RD. 2000. The mouse Spol1 gene is required for meiotic chromosome synapsis. Mol Cell 6: $975-987$.

San Filippo J, Sung P, Klein H. 2008. Mechanism of eukaryotic homologous recombination. Annu Rev Biochem 77: 229257.

Sasanuma H, Hirota K, Fukuda T, Kakusho N, Kugou K, Kawasaki Y, Shibata T, Masai H, Ohta K. 2008. Cdc7dependent phosphorylation of Mer2 facilitates initiation of yeast meiotic recombination. Genes Dev 22: 398-410.

Steiner S, Kohli J, Ludin K. 2010. Functional interactions among members of the meiotic initiation complex in fission yeast. Curr Genet 115: 175-194.

Swanson WJ, Vacquier VD. 2002. The rapid evolution of reproductive proteins. Nat Rev Genet 3: 137-144.

Wan L, Niu H, Futcher B, Zhang C, Shokat KM, Boulton SI, Hollingsworth NM. 2008. Cdc28-Clb5 (CDK-S) and Cdc7Dbf4 (DDK) collaborate to initiate meiotic recombination in yeast. Genes Dev 22: 386-397. 


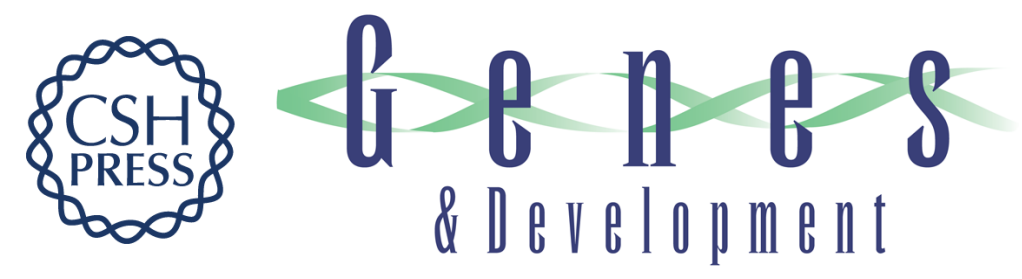

\section{Evolutionary conservation of meiotic DSB proteins: more than just Spo11}

Francesca Cole, Scott Keeney and Maria Jasin

Genes Dev. 2010, 24:

Access the most recent version at doi:10.1101/gad.1944710

\section{Related Content Functional conservation of Mei4 for meiotic DNA double-strand break formation from yeasts to mice \\ Rajeev Kumar, Henri-Marc Bourbon and Bernard de Massy \\ Genes Dev. June , 2010 24: 1266-1280}

References This article cites 45 articles, 13 of which can be accessed free at:

http://genesdev.cshlp.org/content/24/12/1201.full.html\#ref-list-1

Articles cited in:

http://genesdev.cshlp.org/content/24/12/1201.full.html\#related-urls

\section{License}

Email Alerting

Service

Receive free email alerts when new articles cite this article - sign up in the box at the top right corner of the article or click here.

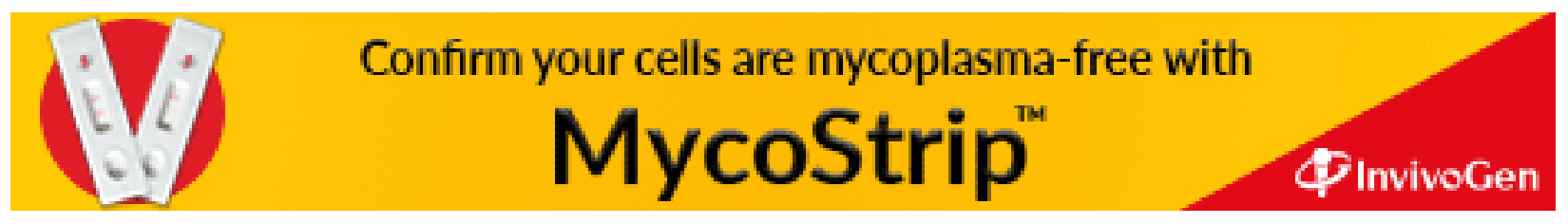

